Research Article

\title{
Boundedness of the Segal-Bargmann Transform on Fractional Hermite-Sobolev Spaces
}

\author{
Hong Rae Cho, Hyunil Choi, and Han-Wool Lee \\ Department of Mathematics, Pusan National University, Busan 609-735, Republic of Korea \\ Correspondence should be addressed to Hong Rae Cho; chohr@pusan.ac.kr \\ Received 21 November 2016; Accepted 4 January 2017; Published 19 January 2017 \\ Academic Editor: Vakhtang M. Kokilashvili
}

Copyright (C) 2017 Hong Rae Cho et al. This is an open access article distributed under the Creative Commons Attribution License, which permits unrestricted use, distribution, and reproduction in any medium, provided the original work is properly cited.

Let $s \in \mathbb{R}$ and $2 \leq p \leq \infty$. We prove that the Segal-Bargmann transform $\mathscr{B}$ is a bounded operator from fractional Hermite-Sobolev spaces $W_{H}^{s, p}\left(\mathbb{R}^{n}\right)$ to fractional Fock-Sobolev spaces $F_{\mathscr{R}}^{s, p}$.

\section{Introduction}

In quantum mechanics, the Schrödinger equation is a partial differential equation that describes how the quantum state of some physical system changes with time. The most famous example is the nonrelativistic Schrödinger equation for a single particle moving in a potential:

$$
\sqrt{-1} \hbar \frac{\partial}{\partial t} \Psi(x, t)=\left[\frac{-\hbar^{2}}{2 m} \Delta+V(x, t)\right] \Psi(x, t),
$$

where $m$ is the particle's mass, $\hbar$ is the Planck constant, $V$ is its potential energy, and $\Psi$ is the wave function.

Let $H$ be the most basic Schrödinger operator in $\mathbb{R}^{n}, n \geq$ 1, the Hermite operator (or the harmonic oscillator):

$$
H=-\Delta+|x|^{2} .
$$

Then the Schrödinger equation can be written by

$$
\sqrt{-1} \frac{\partial \Psi}{\partial t}=H \Psi
$$

This is an important model in quantum mechanics (see, e.g., [1]).

For $s \in \mathbb{R}$, we define the fractional Hermite operator $H^{s}=(-\Delta+|x|)^{s}$ of order $s$. Let $0<p \leq \infty$. The HermiteSobolev space $W_{H}^{s, p}\left(\mathbb{R}^{n}\right)$ of fractional order $s$ is the space of all tempered distributions for which the distribution $H^{s / 2} f$ is given by an $L^{p}$ function on $\mathbb{R}^{n}$.
Let $\mathbb{C}^{n}$ be the complex $n$-space and let $d V$ be the ordinary volume measure on $\mathbb{C}^{n}$. If $z=\left(z_{1}, \ldots, z_{n}\right)$ and $w=$ $\left(w_{1}, \ldots, w_{n}\right)$ are points in $\mathbb{C}^{n}$, we write

$$
\begin{aligned}
z \cdot \bar{w} & =\sum_{j=1}^{n} z_{j} \bar{w}_{j}, \\
|z| & =(z \cdot \bar{z})^{1 / 2} .
\end{aligned}
$$

For any $0<p \leq \infty$ the Fock space $F^{p}$ denotes the space of entire functions $f$ on $\mathbb{C}^{n}$ such that the function $f(z) e^{-(1 / 4)|z|^{2}}$ is in $L^{p}\left(\mathbb{C}^{n}, d V\right)$. We define

$$
\|f\|_{F^{p}}=\left[\left(\frac{p}{4 \pi}\right)^{n} \int_{\mathbb{C}^{n}}\left|f(z) e^{-(1 / 4)|z|^{2}}\right|^{p} d V(z)\right]^{1 / p} .
$$

For $p=\infty$ the norm in $F^{\infty}$ is defined by

$$
\|f\|_{F^{\infty}}=\sup \left\{|f(z)| e^{-(1 / 4)|z|^{2}}: z \in \mathbb{C}^{n}\right\} .
$$

Let

$$
\begin{aligned}
& A_{j} f(z)=2 \frac{\partial}{\partial z_{j}} f(z), \\
& A_{j}^{*} f(z)=z_{j} f(z),
\end{aligned}
$$


Both $A_{j}$ and $A_{j}^{*}$, as defined above, are densely defined linear operators on $F^{P}$ (unbounded though). We consider the radial derivative $\mathscr{R}$ defined by

$$
\mathscr{R}:=\frac{1}{2} \sum_{j=1}^{n}\left(A_{j} A_{j}^{*}+A_{j}^{*} A_{j}\right) .
$$

Let $s$ be a real number and $0<p \leq \infty$. The fractional FockSobolev space $F_{\mathscr{R}}^{s, p}$ of order $s$ is the space of all entire functions for which $\mathscr{R}^{s / 2} f$ is given by an $F^{p}$ function.

The Segal-Bargmann transform $\mathscr{B}$ is defined by

$$
\mathscr{B} f(z)=\frac{1}{\pi^{n / 4}} \int_{\mathbb{R}^{n}} f(x) e^{x \cdot z-(1 / 2)|x|^{2}-(1 / 4) z \cdot z} d V(x),
$$

where $d V(x)$ is the volume measure on $\mathbb{R}^{n}$. It is well-known that the Segal-Bargmann transform is a unitary isomorphism between $L^{2}\left(\mathbb{R}^{n}\right)$ and $F^{2}[2,3]$.

We prove that the radial derivative $\mathscr{R}$ has a parallel behavior to the Hermite operator $H$. In particular, $\mathscr{R}$ is densely defined, positive, self-adjoint and has the discrete spectrum; it generates a diffusion semigroup. Moreover, we show that the Segal-Bargmann transform intertwines fractional Hermite-Sobolev spaces with fractional Fock-Sobolev spaces as follows.

Theorem 1. Let $s \in \mathbb{R}$ and $2 \leq p \leq \infty$. Then the SegalBargmann transform $\mathscr{B}: W_{H}^{s, p}\left(\mathbb{R}^{n}\right) \rightarrow F_{\mathscr{R}}^{s, p}$ is bounded.

\section{Fractional Hermite-Sobolev Spaces}

In one dimension, the Hermite polynomials $H_{k}$ are defined by

$$
H_{k}(x)=e^{x^{2}} \frac{d^{k}}{d x^{k}}\left(e^{-x^{2}}\right), \quad x \in \mathbb{R},
$$

and by normalization we obtain the Hermite functions

$$
h_{k}(x)=\left(\sqrt{\pi} 2^{k} k !\right)^{-1 / 2} e^{-x^{2} / 2}(-1)^{k} H_{k}(x), \quad x \in \mathbb{R} .
$$

Note that

$$
\begin{aligned}
& \left(-\frac{d^{2}}{d x^{2}}+x^{2}\right)\left[e^{-(1 / 2) x^{2}} H_{k}(x)\right] \\
& =(2 k+1)\left[e^{-(1 / 2) x^{2}} H_{k}(x)\right], \quad x \in \mathbb{R} .
\end{aligned}
$$

In higher dimensions, for each multi-index $\alpha=$ $\left(\alpha_{1}, \ldots, \alpha_{n}\right) \in \mathbb{N}_{0}^{n}$, the Hermite functions $h_{\alpha}$ are defined by

$$
h_{\alpha}(x)=\prod_{j=1}^{n} h_{\alpha_{j}}\left(x_{j}\right), \quad x=\left(x_{1}, \ldots, x_{n}\right) \in \mathbb{R}^{n} .
$$

Here, $\mathbb{N}_{0}=\mathbb{N} \cup\{0\}$ is the set of nonnegative integer. By (12), we know that these are the eigenfunctions of the Hermite operator defined in (2). In fact,

$$
H h_{\alpha}=(2|\alpha|+n) h_{\alpha} .
$$

Moreover, $\left\{h_{\alpha}: \alpha \in \mathbb{N}_{0}^{n}\right\}$ is an orthonormal basis for $L^{2}\left(\mathbb{R}^{n}\right)$.
Let $\mathscr{H}$ be the space of finite linear combinations of Hermite functions

$$
f=\sum_{|\alpha| \leq N}\left\langle f, h_{\alpha}\right\rangle h_{\alpha}
$$

where

$$
\left\langle f, h_{\alpha}\right\rangle=\int_{\mathbb{R}^{n}} f(x) h_{\alpha}(x) d V(x) .
$$

The space $\mathscr{H}$ is dense in $L^{2}\left(\mathbb{R}^{n}\right)$, and so, by the orthonormality of the Hermite functions,

$$
\|f\|_{L^{2}\left(\mathbb{R}^{n}\right)}=\left(\sum_{\alpha \in \mathbb{N}_{0}^{n}}\left|\left\langle f, h_{\alpha}\right\rangle\right|^{2}\right)^{1 / 2} .
$$

For $s \in \mathbb{R}$, we define the fractional Hermite operator $H^{s}=$ $(-\Delta+|x|)^{s}$ of order $s$. For $f \in \mathcal{S}\left(\mathbb{R}^{n}\right)$, the Hermite series expansion

$$
\sum_{\alpha \in \mathbb{N}_{0}^{n}}\left\langle f, h_{\alpha}\right\rangle h_{\alpha}
$$

converges to $f$ uniformly in $\mathbb{R}^{n}$ (and also in $L^{2}\left(\mathbb{R}^{n}\right)$ ), since $\left\|h_{\alpha}\right\|_{L^{\infty}\left(\mathbb{R}^{n}\right)} \leq C$, for all $\alpha \in \mathbb{N}_{0}^{n}$, and each $m \in \mathbb{N}$, and we have (see [4])

$$
\left|\left\langle f, h_{\alpha}\right\rangle\right| \leq\left\|H^{m} f\right\|_{L^{2}\left(\mathbb{R}^{n}\right)}(2|\alpha|+n)^{-m} .
$$

Definition 2. Let $s \in \mathbb{R}$ and $f \in \mathcal{S}\left(\mathbb{R}^{n}\right)$. One defines the fractional Hermite operator $H^{s}$ by

$$
H^{s} f=\sum_{\alpha \in \mathbb{N}_{0}^{n}}(2|\alpha|+n)^{s}\left\langle f, h_{\alpha}\right\rangle h_{\alpha} .
$$
[5].

The fractional Hermite operators $H^{s}$ were introduced in

Definition 3. Let $s \in \mathbb{R}$ and $0<p \leq \infty$. The fractional Hermite-Sobolev space $W_{H}^{s, p}\left(\mathbb{R}^{n}\right)$ of order $s$ is the space of all tempered distributions for which the distribution $H^{s / 2} f$ is given by an $L^{p}$ function on $\mathbb{R}^{n}$. The fractional HermiteSobolev norm of order $s$ is defined accordingly,

$$
\|f\|_{W_{H}^{s, p}\left(\mathbb{R}^{n}\right)}=\left\|H^{s / 2} f\right\|_{L^{p}\left(\mathbb{R}^{n}\right)} .
$$

The fractional Hermite-Sobolev spaces $W^{s, p}\left(\mathbb{R}^{n}\right)$ of order $s$ were introduced in [6].

\section{Radial Derivative}

We consider the radial derivative $\mathscr{R}$ defined on

$$
\mathscr{D a m}(\mathscr{R})=\left\{f \in F^{2}: \mathscr{R} f \in F^{2}\right\}
$$

by

$$
\mathscr{R}:=\frac{1}{2} \sum_{j=1}^{n}\left(A_{j} A_{j}^{*}+A_{j}^{*} A_{j}\right),
$$


where

$$
\begin{aligned}
& A_{j} f(z)=2 \frac{\partial}{\partial z_{j}} f(z), \\
& A_{j}^{*} f(z)=z_{j} f(z),
\end{aligned}
$$

$$
1 \leq j \leq n, f \in F^{2} .
$$

We have

$$
\mathscr{R}=2 \sum_{j=1}^{n} z_{j} \frac{\partial}{\partial z_{j}}+n
$$

The following example tells us that $\mathscr{D} a m(\mathscr{R}) \subsetneq F^{2}$. Thus $\mathscr{R}$ is an unbounded operator on $F^{2}$.

Example 4. Let

$$
f(z)=\sum_{k=0}^{\infty} \frac{z_{1}^{k}}{\sqrt{2}^{k}(k+1) \sqrt{k !}} .
$$

Then $f \in F^{2}$, but $\mathscr{R} f \notin F^{2}$.

Proof. Note that

$$
\begin{aligned}
& \|f\|_{F^{2}}^{2} \\
& \quad=\frac{1}{(2 \pi)^{n}} \sum_{k=0}^{\infty} \int_{\mathbb{C}^{n}}\left|\frac{z_{1}^{k}}{\sqrt{2}^{k}(k+1) \sqrt{k !}}\right|^{2} e^{-(1 / 2)|z|^{2}} d V(z) \\
& \quad=\sum_{k=0}^{\infty} \frac{1}{(k+1)^{2}}=\zeta(2)<\infty,
\end{aligned}
$$

where $\zeta(\cdot)$ is the Riemann zeta function. However, we have

$$
\begin{aligned}
\|\mathscr{R} f\|_{F^{2}}^{2} & =\left\|\sum_{k=0}^{\infty} \frac{(2 k+n) z_{1}^{k}}{\sqrt{2}^{k}(k+1) \sqrt{k !}}\right\|_{F^{2}}^{2}=\sum_{k=0}^{\infty} \frac{(2 k+n)^{2}}{(k+1)^{2}} \\
& =\infty .
\end{aligned}
$$

Lemma 5. $\mathscr{R}$ is a positive, self-adjoint operator on $\mathscr{D} a m(\mathscr{R})$.

Proof. Let $\mathscr{P}\left(\mathbb{C}^{n}\right)$ be the set of all holomorphic polynomials on $\mathbb{C}^{n}$. We know that $\mathscr{P}\left(\mathbb{C}^{n}\right)$ is dense in $F^{2}$ and $\mathscr{R}$ is selfadjoint on $\mathscr{P}\left(\mathbb{C}^{n}\right)$. Hence $\mathscr{D a m}(\mathscr{R})$ is the domain of its unique self-adjoint extension.

Note that

$$
\begin{aligned}
\langle f, \mathscr{R} f\rangle_{F^{2}}=2 \sum_{j=1}^{n}\left\|\frac{\partial f}{\partial z_{j}}\right\|_{F^{2}}^{2}+n\|f\|_{F^{2}}^{2} & \geq n\|f\|_{F^{2}}^{2}, \\
\forall f & \in \mathscr{D a m}(\mathscr{R}) .
\end{aligned}
$$

Thus $\mathscr{R}$ is positive.

Lemma 6. $\mathscr{R}$ has the discrete spectrum $\sigma(\mathscr{R})=\{2|\alpha|+n$ : $\left.\alpha \in \mathbb{N}_{0}^{n}\right\}$.
Proof. By (29), we have $\sigma(\mathscr{R}) \subseteq[n, \infty)$.

We define

$$
e_{\alpha}(z)=\frac{z^{\alpha}}{\left\|z^{\alpha}\right\|_{F^{2}}}=\frac{z^{\alpha}}{\sqrt{2^{|\alpha|} \alpha}} .
$$

Then $\left\{e_{\alpha}: \alpha \in \mathbb{N}_{0}^{n}\right\}$ is an orthonormal basis for $F^{2}$. It is easy to see that $\left\{2|\alpha|+n: \alpha \in \mathbb{N}_{0}^{n}\right\}$ is the set of all eigenvalues.

Let $\lambda \in[n, \infty) \backslash\left\{2|\alpha|+n: \alpha \in \mathbb{N}_{0}^{n}\right\}$. First, we show that $\lambda I-\mathscr{R}: \mathscr{D a m}(\mathscr{R}) \rightarrow F^{2}$ is injective and surjective.

Suppose that $(\lambda I-\mathscr{R}) f=(\lambda I-\mathscr{R}) \tilde{f}$. Then

$$
\begin{aligned}
0 & =(\lambda I-\mathscr{R}) f-(\lambda I-\mathscr{R}) \tilde{f} \\
& =\sum_{\alpha \in \mathbb{N}_{0}^{n}}\{\lambda-(2|\alpha|+n)\}\left\langle f-\tilde{f}, e_{\alpha}\right\rangle e_{\alpha} .
\end{aligned}
$$

This implies $f=\tilde{f}$. Thus $\lambda I-\mathscr{R}: \mathscr{D a m}(\mathscr{R}) \rightarrow F^{2}$ is injective.

For $f \in F^{2}$ let

$$
f(z)=\sum_{\alpha \in \mathbb{N}_{0}^{n}} c_{\alpha} e_{\alpha}(z)
$$

be the orthonormal decomposition of $f$. We define

$$
g=\frac{1}{\lambda} f+\frac{1}{\lambda} \sum_{\alpha \in \mathbb{N}_{0}^{n}} \frac{2|\alpha|+n}{\lambda-(2|\alpha|+n)} c_{\alpha} e_{\alpha}(z) .
$$

Since

$$
\varphi_{N}=\sum_{|\alpha|=0}^{N} \frac{2|\alpha|+n}{\lambda-(2|\alpha|+n)} c_{\alpha} e_{\alpha}(z)
$$

is a Cauchy sequence in $F^{2}$, the series in (33) converges in $F^{2}$. Hence

$$
g=\frac{1}{\lambda} f+\frac{1}{\lambda} \sum_{|\alpha|=0}^{\infty} \frac{2|\alpha|+n}{\lambda-(2|\alpha|+n)} c_{\alpha} e_{\alpha}(z)
$$

is a well-defined element of $F^{2}$ and it satisfies $(\lambda I-\mathscr{R}) g=f$. This means that $\lambda I-\mathscr{R}: \mathscr{D} a m(\mathscr{R}) \rightarrow F^{2}$ is surjective.

Moreover,

$$
\begin{aligned}
\left\|(\lambda I-\mathscr{R})^{-1} f\right\|_{F^{2}} & \leq \frac{1}{\lambda}\|f\|_{F^{2}}+\frac{1}{\lambda} \beta\|f\|_{F^{2}} \\
& =\frac{1}{\lambda}(1+\beta)\|f\|_{F^{2}},
\end{aligned}
$$

where $\beta=\sup _{\alpha \in \mathbb{N}_{0}^{n}}|(2|\alpha|+n) /(\lambda-(2|\alpha|+n))|$. Hence $(\lambda I-\mathscr{R})^{-1}$ is bounded and so $\sigma(\mathscr{R})=\left\{2|\alpha|+n: \alpha \in \mathbb{N}_{0}^{n}\right\}$.

For $f \in F^{2}$ let

$$
f(z)=\sum_{\alpha \in \mathbb{N}_{0}^{n}} c_{\alpha} e_{\alpha}(z)
$$

be the orthonormal decomposition of $f$. Associated with the operator $\mathscr{R}$ is a semigroup $\left\{B_{t}\right\}_{t \geq 0}$ defined by the expansion

$$
B_{t} f(z)=\sum_{\alpha \in \mathbb{N}_{0}^{n}} e^{-(2|\alpha|+n) t} c_{\alpha} e_{\alpha}(z) .
$$


We can check that $u(z, t):=B_{t} f(z)$ is the solution of the heattype equation:

$$
\begin{array}{cc}
\left(\partial_{t}+\mathscr{R}\right) u=0 & \text { on } \mathbb{C}^{n} \times(0, \infty), \\
u(\cdot, 0)=f & \text { on } \mathbb{C}^{n} .
\end{array}
$$

It is easy to see that

$$
\left\|B_{t} f\right\|_{F^{2}}^{2} \leq e^{-2 n t}\|f\|_{F^{2}}^{2} .
$$

Thus $B_{t}$ is contractive.

Proposition 7. $\left\{B_{t}\right\}_{t \geq 0}$ is a strongly continuous semigroup.

Proof. We note that

$$
\begin{aligned}
\left\|B_{t} f-f\right\|_{F^{2}}^{2} & =\sum_{\alpha \in \mathbb{N}_{0}^{n}}\left|e^{-(2|\alpha|+n) t}-1\right|^{2}\left|c_{\alpha}\right|^{2} \\
& =\sum_{k=0}^{\infty}\left|e^{-(2 k+n) t}-1\right|^{2} \sum_{|\alpha|=k}\left|c_{\alpha}\right|^{2} .
\end{aligned}
$$

For $k \in \mathbb{N}_{0}$ and $X \subset \mathbb{N}_{0}$ we define $\delta_{k}(X)$ by

$$
\delta_{k}(X)= \begin{cases}1, & \text { if } k \in X, \\ 0, & \text { if } k \notin X .\end{cases}
$$

Then

$$
\begin{aligned}
\lim _{t \rightarrow 0^{+}}\left\|B_{t} f-f\right\|_{F^{2}}^{2} & =\lim _{t \rightarrow 0^{+}} \sum_{k=0}^{\infty}\left|e^{-(2 k+n) t}-1\right|^{2} \sum_{|\alpha|=k}\left|c_{\alpha}\right|^{2} \\
& =\lim _{t \rightarrow 0^{+}} \int_{0}^{\infty}\left|e^{-(2 \lambda+n) t}-1\right|^{2} d \nu(\lambda),
\end{aligned}
$$

where $v$ is a discrete measure defined by

$$
\nu=\sum_{k=0}^{\infty}\left(\sum_{|\alpha|=k}\left|c_{\alpha}\right|^{2}\right) \delta_{k} .
$$

By Lebesgue dominate convergence theorem, we have

$$
\begin{aligned}
\lim _{t \rightarrow 0^{+}}\left\|B_{t} f-f\right\|_{F^{2}}^{2} & =\int_{0}^{\infty} \lim _{t \rightarrow 0^{+}}\left|e^{-(2 \lambda+n) t}-1\right|^{2} d \nu(\lambda) \\
& =0
\end{aligned}
$$

Hence $\left\{B_{t}\right\}_{t \geq 0}$ is a strongly continuous semigroup.

Proposition 8. $-\mathscr{R}$ is the infinitesimal generator of $\left\{B_{t}\right\}_{t \geq 0}$. That is,

$$
\lim _{t \rightarrow 0^{+}} \frac{B_{t} f-f}{t}=-\mathscr{R} f .
$$

Proof. By using the previous discrete measure $v$, it follows that

$$
\begin{aligned}
& \left\|\frac{B_{t} f-f}{t}-(-\mathscr{R} f)\right\|_{F^{2}}^{2} \\
& \quad=\int_{0}^{\infty}\left|\frac{e^{-(2 \lambda+n) t}-1}{t}+(2 \lambda+n)\right|^{2} d \nu(\lambda) .
\end{aligned}
$$

Taking limit on both sides and by Lebesgue dominate convergence theorem,

$$
\begin{aligned}
\lim _{t \rightarrow 0^{+}} & \left\|\frac{B_{t} f-f}{t}-(-\mathscr{R} f)\right\|_{F^{2}}^{2} \\
& =\lim _{t \rightarrow 0^{+}} \int_{0}^{\infty}\left|\frac{e^{-(2 \lambda+n) t}-1}{t}+(2 \lambda+n)\right|^{2} d \nu(\lambda) \\
& =\int_{0}^{\infty} \lim _{t \rightarrow 0^{+}}\left|\frac{e^{-(2 \lambda+n) t}-1}{t}+(2 \lambda+n)\right|^{2} d \nu(\lambda)=0 .
\end{aligned}
$$

Thus we get the result.

By Proposition 8, we have

$$
B_{t}=e^{-t \mathscr{R}}
$$

\section{Fractional Fock-Sobolev Spaces}

Since $\mathscr{R}$ has discrete spectrum $\left\{2|\alpha|+n: \alpha \in \mathbb{N}_{0}^{n}\right\}$, by using the spectral theorem, we define the fractional radial derivative $\mathscr{R}^{s}$ for $s \in \mathbb{R}$ as follows.

Definition 9. Let $s \in \mathbb{R}$. For $f \in F^{2}$ let

$$
f(z)=\sum_{\alpha \in \mathbb{N}_{0}^{n}} c_{\alpha} e_{\alpha}(z)
$$

be the orthonormal decomposition of $f$. By the spectral theorem, $\mathscr{R}^{s}$ is given by

$$
\begin{aligned}
\mathscr{R}^{s} f(z)=\sum_{\alpha \in \mathbb{N}_{0}^{n}}(2|\alpha|+n)^{s} c_{\alpha} e_{\alpha}(z), & \\
& f \in \operatorname{Dam}\left(\mathscr{R}^{s}\right) .
\end{aligned}
$$

Definition 10. Let $s$ be a real number and $0<p \leq \infty$. The fractional Fock-Sobolev space $F_{\mathscr{R}}^{s, p}$ of order $s$ is the space of all entire functions for which $\mathscr{R}^{s / 2} f$ is given by an $F^{p}$ function. The fractional Fock-Sobolev norm of $f$ of order $s$ is defined accordingly,

$$
\|f\|_{F_{\mathscr{R}}^{s, p}}=\left\|\mathscr{R}^{s / 2} f\right\|_{F^{p}} .
$$

We refer the reader to [7-10] for other Fock-Sobolev spaces.

\section{5. $L^{p}$-Boundedness of the Segal-Bargmann Transform}

The Hermite operator $H$ is self-adjoint on the set of infinitely differentiable functions with compact support $C_{c}^{\infty}\left(\mathbb{R}^{n}\right)$, and it can be factorized as

$$
H=\frac{1}{2} \sum_{j=1}^{n}\left(a_{j} a_{j}^{\dagger}+a_{j}^{\dagger} a_{j}\right),
$$


where

$$
\begin{aligned}
& a_{j}=\frac{\partial}{\partial x_{j}}+x_{j}, \\
& a_{j}^{\dagger}=-\frac{\partial}{\partial x_{j}}+x_{j},
\end{aligned}
$$

$$
1 \leq j \leq n .
$$

Lemma 11. For each $j=1, \ldots, n$, one has

$$
\begin{aligned}
& \mathscr{B}\left(a_{j} f\right)=A_{j} \mathscr{B}(f), \\
& \mathscr{B}\left(a_{j}^{\dagger} f\right)=A_{j}^{*} \mathscr{B}(f) .
\end{aligned}
$$

Proof. Let $f \in C_{c}^{\infty}\left(\mathbb{R}^{n}\right)$. By the integration by parts, we have

$$
\begin{aligned}
\mathscr{B} & \left(\frac{\partial}{\partial x_{j}} f\right)(z) \\
\quad & =\frac{1}{\pi^{n / 4}} \int_{\mathbb{R}^{n}} \frac{\partial f}{\partial x_{j}}(x) e^{x \cdot z-(1 / 2)|x|^{2}-(1 / 4) z \cdot z} d V(x) \\
& =-z_{j} \mathscr{B}(f)+\mathscr{B}\left(x_{j} f\right) .
\end{aligned}
$$

This gives

$$
\mathscr{B}\left(a_{j}^{\dagger} f\right)=A_{j}^{*} \mathscr{B}(f) .
$$

We differentiate

$$
\mathscr{B} f(z)=\frac{1}{\pi^{n / 4}} \int_{\mathbb{R}^{n}} f(x) e^{x \cdot z-(1 / 2)|x|^{2}-(1 / 4) z \cdot z} d V(x)
$$

under the integral sign to obtain

$$
\begin{aligned}
A_{j} \mathscr{B} f(z)=\frac{1}{\pi^{n / 4}} \\
\quad \cdot \int_{\mathbb{R}^{n}}\left(2 x_{j}-z_{j}\right) f(x) e^{x \cdot z-(1 / 2)|x|^{2}-(1 / 4) z \cdot z} d V(x) .
\end{aligned}
$$

This gives

$$
A_{j} \mathscr{B}(f)=2 \mathscr{B}\left(x_{j} f\right)-A_{j}^{*} \mathscr{B}(f) .
$$

By (57) and (60), it follows that

$$
A_{j} \mathscr{B}(f)=\mathscr{B}\left(a_{j} f\right) .
$$

Corollary 12. Consider

$$
\mathscr{B} H=\mathscr{R} \mathscr{B} .
$$

Proof. By Lemma 11, we have

$$
\mathscr{B}(H f)=\frac{1}{2} \sum_{j=1}^{n}\left(A_{j} A_{j}^{*}+A_{j}^{*} A_{j}\right) \mathscr{B}(f)=\mathscr{R} \mathscr{B} .
$$

Proposition 13. Let $s \in \mathbb{R}$. Then

$$
\mathscr{B} H^{s}=\mathscr{R}^{s} \mathscr{B} .
$$

Proof. We define

$$
e_{\alpha}(z)=\frac{z^{\alpha}}{\left\|z^{\alpha}\right\|_{F^{2}}} .
$$

Then $\left\{e_{\alpha}: \alpha \in \mathbb{N}_{0}^{n}\right\}$ is an orthonormal basis for $F^{2}$ and $\mathscr{B}\left(h_{\alpha}\right)=e_{\alpha}$. For $f \in \mathcal{S}\left(\mathbb{R}^{n}\right)$ we have

$$
H^{s} f=\sum_{\alpha \in \mathbb{N}_{0}^{n}}(2|\alpha|+n)^{s}\left\langle f, h_{\alpha}\right\rangle h_{\alpha}
$$

and so

$$
\mathscr{B}\left(H^{s} f\right)=\sum_{\alpha \in \mathbb{N}_{0}^{n}}(2|\alpha|+n)^{s}\left\langle f, h_{\alpha}\right\rangle e_{\alpha} .
$$

Since $\mathscr{B}$ is a unitary isomorphism, we have $\left\langle f, h_{\alpha}\right\rangle=$ $\left\langle\mathscr{B}(f), e_{\alpha}\right\rangle$. Hence

$$
\begin{aligned}
\mathscr{B}\left(H^{s} f\right) & =\sum_{\alpha \in \mathbb{N}_{0}^{n}}(2|\alpha|+n)^{s}\left\langle\mathscr{B}(f), e_{\alpha}\right\rangle e_{\alpha} \\
& =\mathscr{R}^{s} \mathscr{B}(f) .
\end{aligned}
$$

Thus we get the result.

We consider the mapping property of the SegalBargmann transform $\mathscr{B}$ as a map from $L^{p}\left(\mathbb{R}^{n}\right)$ to $F^{p}$ for $p \in[2, \infty]$. Note that one-dimensional case is in [11].

Theorem 14. Consider

$$
\|\mathscr{B} f\|_{F^{\infty}} \leq(4 \pi)^{n / 4}\|f\|_{L^{\infty}\left(\mathbb{R}^{n}\right)} .
$$

Proof. We have

$$
\begin{aligned}
& |\mathscr{B} f(z)| \leq \frac{1}{\pi^{n / 4}} e^{|z|^{2} / 4} \sup _{x \in \mathbb{R}^{n}}|f(x)| \\
& \cdot \int_{\mathbb{R}^{n}} e^{\operatorname{Re}(z \cdot x)-(1 / 2)|x|^{2}-(1 / 4) \operatorname{Re}(z \cdot z)-|z|^{2} / 4} d V(x) .
\end{aligned}
$$

Note that

$$
|\operatorname{Re}(z)|^{2}=\frac{1}{2}\left\{|z|^{2}+\operatorname{Re}(z \cdot z)\right\} .
$$

Hence

$$
\begin{aligned}
\operatorname{Re} & (z \cdot x)-\frac{1}{2}|x|^{2}-\frac{1}{4} \operatorname{Re}(z \cdot z)-\frac{|z|^{2}}{4} \\
& =\operatorname{Re}(z \cdot x)-\frac{1}{2}|x|^{2}-\frac{1}{2}|\operatorname{Re}(z)|^{2} \\
& =-\frac{1}{2}|\operatorname{Re}(z)-x|^{2}
\end{aligned}
$$


and so

$$
\begin{aligned}
& |\mathscr{B} f(z)| \\
& \leq \frac{1}{\pi^{n / 4}} e^{|z|^{2} / 4} \sup _{x \in \mathbb{R}^{n}}|f(x)| \int_{\mathbb{R}^{n}} e^{-(1 / 2)|\operatorname{Re}(z)-x|^{2}} d V(x) \\
& \quad=(4 \pi)^{n / 4} e^{|z|^{2} / 4} \sup _{x \in \mathbb{R}^{n}}|f(x)| .
\end{aligned}
$$

Thus we get the result.

The following Stein-Weiss interpolation theorem is wellknown. See, for example, [3, 12].

Lemma 15. Let $w, w_{0}$, and $w_{1}$ be positive weight functions on a measure space $(X, d \lambda)$. If $1 \leq p_{0} \leq p_{1} \leq \infty$ and $0 \leq \theta \leq 1$, then

$$
\left[L^{p_{0}}\left(X, w_{0} d \lambda\right), L^{p_{1}}\left(X, w_{1} d \lambda\right)\right]_{\theta}=L^{p}(X, w d \lambda)
$$

with equal norms, where

$$
\begin{aligned}
\frac{1}{p} & =\frac{1-\theta}{p_{0}}+\frac{\theta}{p_{1}}, \\
w^{1 / p} & =w_{0}^{(1-\theta) / p_{0}} w_{1}^{\theta / p_{1}} .
\end{aligned}
$$

Theorem 16. Let $2 \leq p \leq \infty$. There exists $C>0$ such that

$$
\|\mathscr{B} f\|_{F^{p}} \leq C\|f\|_{L^{p}\left(\mathbb{R}^{n}\right)} .
$$

Proof. The $L^{2}$-boundedness is followed by the unitary isomorphism of the Segal-Bargmann transform. In Theorem 14, we proved the $L^{\infty}$-boundedness of the Segal-Bargmann transform. By Lemma 15, we have the required result.

By Proposition 13 and Theorem 16, we have the following result.

Theorem 17. Let $s \in \mathbb{R}$ and $2 \leq p \leq \infty$. Then the SegalBargmann transform $\mathscr{B}: W_{H}^{s, p}\left(\mathbb{R}^{n}\right) \rightarrow F_{\mathscr{R}}^{s, p}$ is bounded.

\section{Disclosure}

An earlier version of this work was presented as an abstract at the International Conference on the 70th Anniversary of the Korean Mathematical Society, 2016.

\section{Competing Interests}

The authors declare that there is no conflict of interests regarding the publication of this paper.

\section{Acknowledgments}

The authors would like to thank the referee for his/her valuable remarks and suggestions. The first author was supported by NRF of Korea (NRF-2016R1D1A1B03933740).

\section{References}

[1] R. P. Feynman and A. R. Hibbs, Quantum Mechanics and Path Integrals, McGraw-Hill, Maidenhead, UK, 1965.

[2] B. Hall, Quamtum Theory for Mathematicians, vol. 267, Springer, New York, NY, USA, 2013.

[3] K. Zhu, Analysis on Fock Spaces, vol. 263 of Graduate Texts in Mathematics, Springer, New York, NY, USA, 2012.

[4] P. R. Stinga and J. L. Torrea, "Regularity theory for the fractional harmonic oscillator," Journal of Functional Analysis, vol. 260, no. 10, pp. 3097-3131, 2011.

[5] P. R. Stinga and J. L. Torrea, "Extension problem and Harnack's inequality for some fractional operators," Communications in Partial Differential Equations, vol. 35, no. 11, pp. 2092-2122, 2010.

[6] B. Bongioanni and J. L. Torrea, "Sobolev spaces associated to the harmonic oscillator," Indian Academy of Sciences. Proceedings. Mathematical Sciences, vol. 116, no. 3, pp. 337-360, 2006.

[7] H. R. Cho, B. R. Choe, and H. Koo, "Fock-Sobolev spaces of fractional order," Potential Analysis, vol. 43, no. 2, pp. 199-240, 2015.

[8] H. R. Cho and K. Zhu, "Fock-Sobolev spaces and their Carleson measures," Journal of Functional Analysis, vol. 263, no. 8, pp. 2483-2506, 2012.

[9] B. C. Hall and W. Lewkeeratiyutkul, "Holomorphic Sobolev spaces and the generalized SEGal-Bargmann transform," Journal of Functional Analysis, vol. 217, no. 1, pp. 192-220, 2004.

[10] R. Radha and S. Thangavelu, "Holomorphic Sobolev spaces, HERmite and special HERmite semigroups and a Paley-Wiener theorem for the windowed Fourier transform," Journal of Mathematical Analysis and Applications, vol. 354, no. 2, pp. 564574, 2009.

[11] K. Zhu, "Towards a dictionary for the Bargmann transform," https://arxiv.org/abs/1506.06326.

[12] E. M. Stein and G. Weiss, "Interpolation of operators with change of measures," Transactions of the American Mathematical Society, vol. 87, pp. 159-172, 1958. 


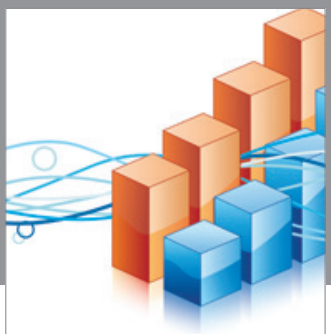

Advances in

Operations Research

vatem alat4

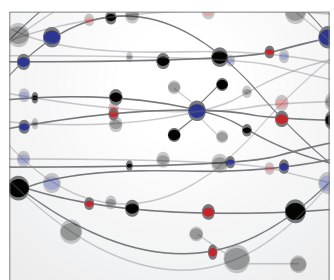

\section{The Scientific} World Journal
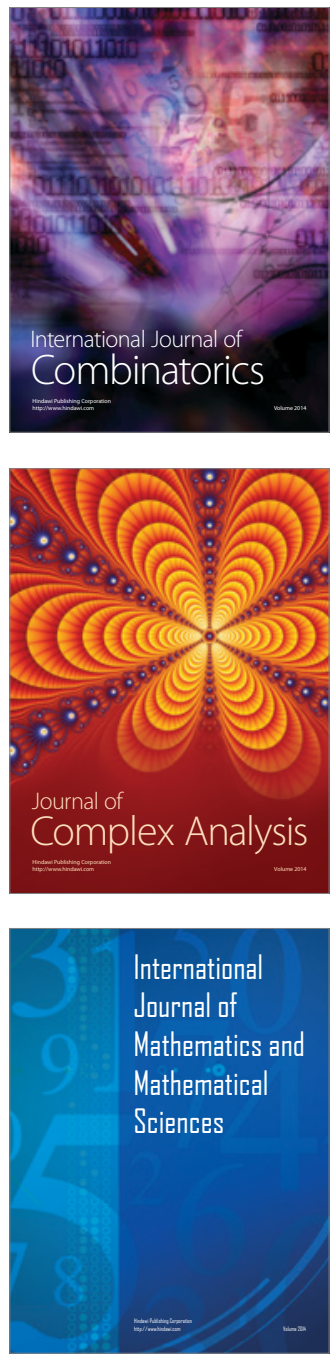
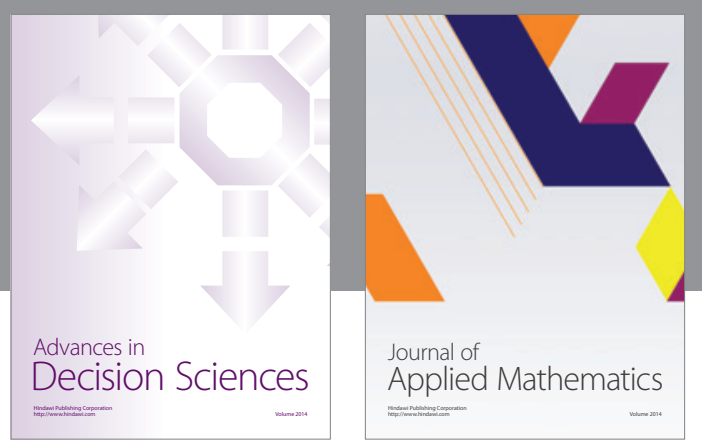

Algebra

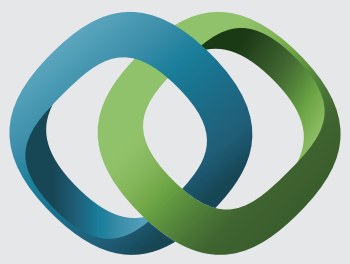

\section{Hindawi}

Submit your manuscripts at

https://www.hindawi.com
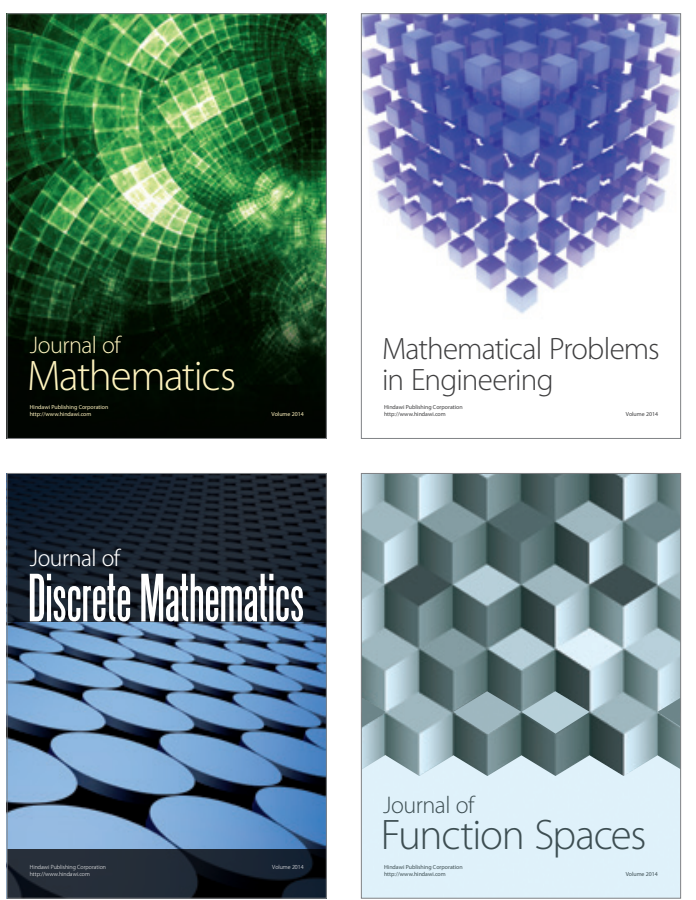

Mathematical Problems in Engineering
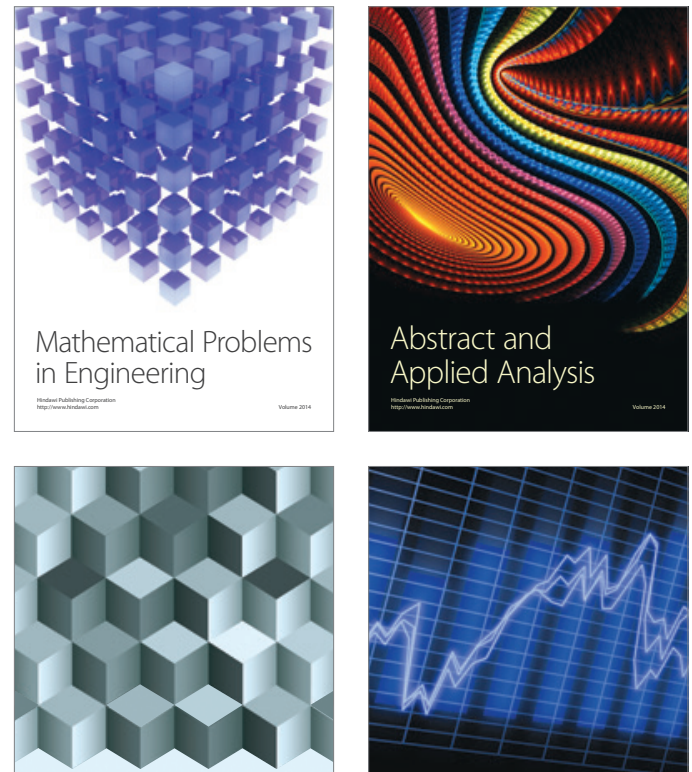

Journal of

Function Spaces

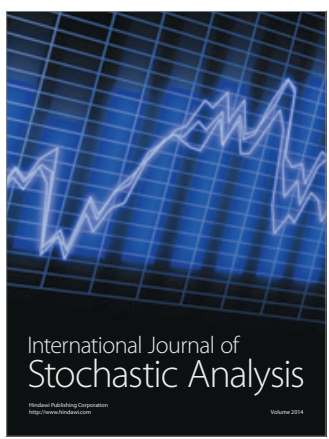

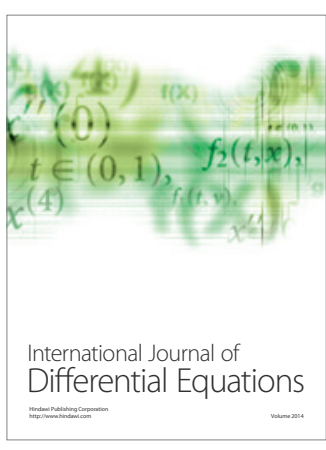
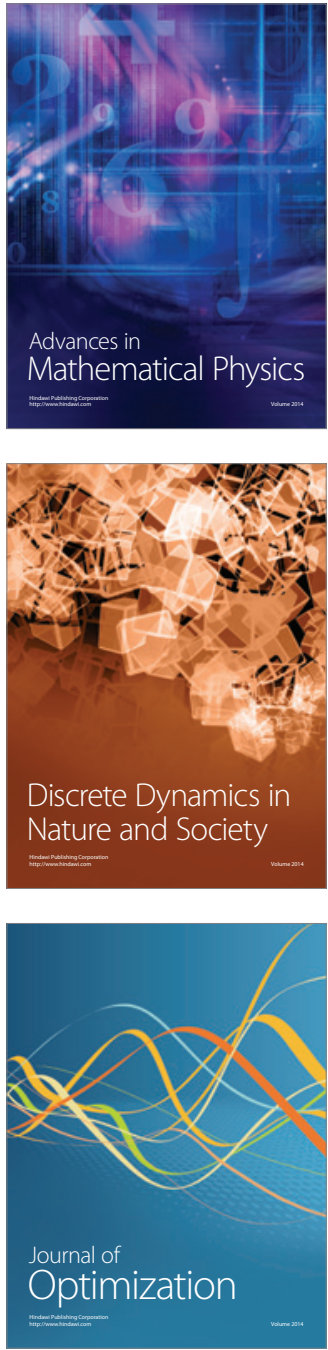